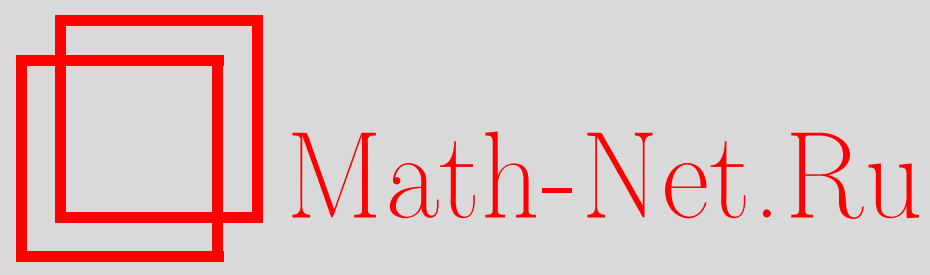

В. А. Копытцев, О пороговом эффекте для среднего числа решений системы случайных уравнений, Матем. вопр. криптогр., 2019, том 10, выпуск 3, 67-80

DOI: https://doi.org/10.4213/mvk300

Использование Общероссийского математического портала Math-Net.Ru подразумевает, что вы прочитали и согласны с пользовательским соглашением http://www . mathnet.ru/rus/agreement

Параметры загрузки:

IP : 54.162 .85 .209

26 апреля 2023 г., 03:41:41

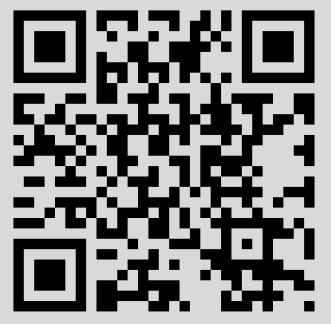


МАТЕМАТИЧЕСКИЕ ВОПРОСЫ КРИПТОГРАФИИ

2019 T. 10 № 3 C. $67-80$

УДК 519.212.2

DOI https://doi.org/10.4213/mvk300

\section{О пороговом эффекте для среднего числа решений системы случайных уравнений}

В. А. Копытцев

Академия криптографии Российской Федерачии, Москва

Получено 10.I.2019

Аннотация. Исследуется система со случайным выбором переменных в каждом уравнении. Доказывается теорема о пороговом эффекте для математического ожидания числа решений.

Ключевые слова: система случайных уравнений, число решений, математическое ожидание, пороговый эффект

On a threshold effect for the mean number of solutions of systems of random equations

V. A. Kopyttsev

Academy of Cryptography of the Russian Federation, Moscow

Abstract. The system with a random sampling of variables in each equation is investigated. The theorem on a threshold effect for the mean number of solutions is proved.

Keywords: system of random equations, number of solutions, mathematical expectation, threshold effect 


\section{1. Введение}

Пусть $\xi$ - число решений случайной системы с числом уравнений $T$ и числом неизвестных $n$. Математическое ожидание $\mathbf{E} \xi$ обладает пороговым эффектом при $T, n \rightarrow \infty$, если существует такое число $\gamma^{*}>0$, что $\mathbf{E} \xi \rightarrow 0$ при $T / n \rightarrow \gamma>\gamma^{*}$ и $\mathbf{E} \xi \rightarrow \infty$ при $T / n \rightarrow \gamma<\gamma^{*}$.

Пороговый эффект для различных характеристик систем случайных уравнений исследовался в ряде работ (см., например, [1-4]). В данной работе исследуется система случайных уравнений с независимыми левыми и правыми частями и случайным выбором неизвестных, принимающих значения в произвольном конечном множестве.

Пусть $V_{q}=\{0,1, \ldots, q-1\}, q \geqslant 2$, функция $\varphi\left(y_{1}, \ldots, y_{d}\right)$ задана на множестве $V_{q}^{d}$ всех $d$-мерных векторов с координатами $y_{1}, \ldots, y_{d} \in V_{q}$ и принимает значения в некотором множестве $A_{\varphi}$.

Обозначим $\Sigma_{\varphi}$ группу инерции функции $\varphi$ в симметрической группе $S_{d}$ перестановок аргументов, определяемую условием

$$
\sigma \in \Sigma_{\varphi} \Leftrightarrow \varphi\left(y_{\sigma(1)}, \ldots, y_{\sigma(d)}\right) \equiv \varphi\left(y_{1}, \ldots, y_{d}\right) .
$$

Рассмотрим случайное уравнение

$$
\varphi\left(x_{s_{1}}, \ldots, x_{s_{d}}\right)=a
$$

относительно переменных $x_{1}, \ldots, x_{n} \in\{0, \ldots, q-1\}$, где индексы $s_{1}, \ldots, s_{d}$ образуют случайную выборку без возвращения из множества $\{1, \ldots, n\}$. Будем предполагать, что упорядоченные выборки $\left(s_{\sigma(1)}, \ldots, s_{\sigma(d)}\right)$, $\sigma \in \Sigma_{\varphi}$, не различаются и объединяются в один исход. На множестве $\Omega=\{\omega\}$ возможных исходов (классов эквивалентных левых частей уравнения) задано равномерное распределение вероятностей

$$
P(\omega)=\left[\left(\begin{array}{c}
n \\
d
\end{array}\right) \frac{d !}{\left|\Sigma_{\varphi}\right|}\right]^{-1} \quad \forall \omega \in \Omega .
$$

Правая часть $a \in A_{\varphi}$ уравнения (1) является случайной величиной и не зависит от левой части. Положим

$$
p(\alpha)=\mathbf{P}\{a=\alpha\}, \quad \alpha \in A_{\varphi} .
$$

Пусть случайная система уравнений

$$
\varphi\left(x_{s_{1}(t)}, \ldots, x_{s_{d}(t)}\right)=a_{t}, \quad t=1, \ldots, T,
$$

состоит из независимо выбранных уравнений вида (1). 
Предположим теперь, что задан конечный набор функций

$$
\Phi=\left\{\varphi_{1}, \varphi_{2}, \ldots, \varphi_{R}\right\},
$$

где

$$
\begin{gathered}
\varphi_{r}=\varphi_{r}\left(y_{1}, \ldots, y_{d(r)}\right), \\
y_{1}, \ldots, y_{d(r)} \in V_{q}, r=1, \ldots, R .
\end{gathered}
$$

Рассмотрим систему уравнений

$$
\begin{gathered}
\varphi_{r}\left(x_{s_{1}(t, r)}, \ldots, x_{s_{d(r)}(t, r)}\right)=a_{t, r}, \\
t=1, \ldots, T(r), r=1, \ldots, R
\end{gathered}
$$

составленную из независимо выбранных подсистем вида (3), соответствующих функциям $\varphi_{1}, \ldots, \varphi_{R}$. Распределения величин $a_{t, r}-$ правых частей системы (4) - могут зависеть от номера $r$.

Будем исследовать свойства математического ожидания числа решений системы уравнений (4) в предположении, что $T(r) \rightarrow \infty, R$ фиксировано и

$$
\frac{T(r)}{T} \rightarrow \tau(r), \quad r=1, \ldots, R,
$$

где $T=T(1)+\ldots+T(R)$, а $\tau(r)$ - постоянные величины.

\section{2. Теорема о пороговом эффекте}

Введем в рассмотрение величины

$$
L_{\varphi_{r}}\left(\alpha ; d_{0}, \ldots, d_{q-1}\right)=\left|\left\{y \in W\left(d_{0}, \ldots, d_{q-1}\right): \varphi_{r}(y)=\alpha \in A_{\varphi_{r}}\right\}\right|,
$$

где $W\left(d_{0}, \ldots, d_{q-1}\right)$ - множество всех векторов $y=\left(y_{1}, \ldots, y_{d(r)}\right)$, в которых $d_{i}$ координат принимают значение $i \in V_{q}, d_{0}+\ldots+d_{q-1}=d(r)$. Положим

$$
L_{\varphi_{r}}\left(d_{0}, \ldots, d_{q-1}\right)=\sum_{\alpha \in A_{\varphi_{r}}} P_{r}(\alpha) L_{\varphi_{r}}\left(\alpha ; d_{0}, \ldots, d_{q-1}\right),
$$

где $P_{r}(\alpha)=\mathbf{P}\left\{a_{t, r}=\alpha\right\}$.

Для формулировки теоремы нам понадобится следующее условие.

У с л о в и е А. Каждая функция $\varphi_{r}=\varphi_{r}\left(y_{1}, \ldots, y_{d(r)}\right), r=1, \ldots, R$, принимает все значения в множестве $A_{\varphi_{r}},\left|A_{\varphi_{r}}\right| \geqslant 2$, и при всех $\alpha \in A_{\varphi_{r}}$, $t=1, \ldots, T(r)$

$$
P_{r}(\alpha)=\mathbf{P}\left\{a_{t, r}=\alpha\right\}>0 .
$$


Заметим, что при условии А выполнены неравенства

$$
L_{\varphi_{r}}\left(d_{0}, \ldots, d_{q-1}\right)>0
$$

при всех $d_{0}, \ldots, d_{q-1} \geqslant 0, d_{0}+\ldots+d_{q-1}=d(r)$.

В области значений переменных $z_{0}, \ldots, z_{q-1}$, заданной условиями

$$
0 \leqslant z_{i}, z_{0}+\ldots+z_{q-1}=1
$$

определим функцию

$$
f\left(z_{0}, \ldots, z_{q-1}\right)=\sum_{r=1}^{R} \tau(r) \ln \left[\sum_{\substack{d_{0}, \ldots, d_{q-1} \\ d_{0}+\ldots+d_{q-1}=d(r)}} \prod_{i=0}^{q-1}\left(z_{i}\right)^{d_{i}} L_{\varphi_{r}}\left(d_{0}, \ldots, d_{q-1}\right)\right]
$$

где $\left(z_{i}\right)^{d_{i}}=1$ при $d_{i}=0$. В силу (8) функция (10) принимает конечные значения во всех точках области (9). Определим также функцию

$$
H\left(z_{0}, \ldots, z_{q-1}\right)=\sum_{i=0}^{q-1} z_{i} \ln z_{i} .
$$

Лемма. Пусть выполнено условие $\boldsymbol{A}$. Тогда существует конечнал верхняя гранииа

$$
\begin{gathered}
\gamma^{*}=\gamma^{*}\left(\varphi_{1}, \ldots, \varphi_{R} ; \tau(1), \ldots, \tau(R) ; P_{1}(\alpha), \ldots, P_{R}(\alpha)\right)= \\
=\sup _{\substack{0 \leqslant z_{0}, \ldots, 0 \leqslant z_{q-1} \\
z_{0}+\ldots+z_{q-1}=1}} \frac{H\left(z_{0}, \ldots, z_{q-1}\right)}{f\left(z_{0}, \ldots, z_{q-1}\right)},
\end{gathered}
$$

зависящая от функиий $\varphi_{r}$, чисел $\tau(r)$ и распределений $P_{r}(\alpha), \alpha \in A_{\varphi_{r}}$, $r=1, \ldots, R$.

Доказательство. Из определения величин (6) вытекает, что

$$
L_{\varphi_{r}}\left(\alpha ; d_{0}, \ldots, d_{q-1}\right) \leqslant\left|W\left(d_{0}, \ldots, d_{q-1}\right)\right|=\frac{d(r) !}{d_{0} ! \cdot \ldots \cdot d_{q-1} !},
$$

при этом равенство в соотношении (13) не может достигаться одновременно при различных $\alpha$. По условию $\mathbf{A}$ все $P_{r}(\alpha)<1$. Поэтому при любом наборе $d_{0}, \ldots, d_{q-1}, d_{0}+\ldots+d_{q-1}=d(r)$, выполнено неравенство

$$
L_{\varphi_{r}}\left(d_{0}, \ldots, d_{q-1}\right)<\frac{d(r) !}{d_{0} ! \cdot \ldots \cdot d_{q-1} !} .
$$


Отсюда вытекает, что

$$
\sum_{\substack{d_{0}, \ldots, d_{q-1} \\ d_{0}+\ldots+d_{q-1}=d(r)}} \prod_{i=0}^{q-1}\left(z_{i}\right)^{d_{i}} L_{\varphi_{r}}\left(d_{0}, \ldots, d_{q-1}\right) \leqslant 1-\delta_{\varphi_{r}}
$$

при некоторой постоянной $\delta_{\varphi_{r}}, 0<\delta_{\varphi_{r}}<1$. Следовательно,

$$
f\left(z_{0}, \ldots, z_{q-1}\right) \leqslant \sum_{r=1}^{R} \tau(r) \ln \left(1-\delta_{\varphi_{r}}\right)<0
$$

и в области (9) существует конечная верхняя граница (12). Лемма 1 доказана.

Пусть $\mathbf{E} \xi$ - математическое ожидание числа решений системы уравнений (4). Положим

$$
T=T(1)+\ldots+T(R) .
$$

Теорема. Пусть $n, T \rightarrow \infty$, въполнень условие $\mathbf{A}$ и условие (5). Тогда

$$
\begin{gathered}
\mathbf{E} \xi \rightarrow 0, \text { если } \underline{\lim (T / n)>\gamma^{*},} \\
\mathbf{E} \xi \rightarrow \infty, \text { если } \overline{\lim }(T / n)<\gamma^{*},
\end{gathered}
$$

где $\gamma^{*}$ - постоянная величина, заданная формулой (12).

Замечание 1. В [5] исследовалась система уравнений, левая часть которой определяется так же, как в системе (4), а правая является результатом подстановки в левую часть некоторого вектора $x^{0}=\left(x_{1}^{0}, \ldots, x_{n}^{0}\right)$. Таким образом, рассмотренная в [5] система является заведомо совместной, в то время как при наличии порогового эффекта (для среднего числа решений) вероятность совместности системы (3) стремится к нулю, если $T / n \rightarrow \gamma>\gamma^{*}$.

Замечание 2. Условие $\mathbf{A}$ в теореме 1 является существенным. Пусть, например, $R=1, \tau_{1}=1, \varphi_{1}=\varphi=y_{1} \vee y_{2}$. Положим $P(0)=\mathbf{P}\left\{a_{t}=0\right\}=1$. В этом случае система уравнений является заведомо совместной, так как всегда имеет решение $x^{0}=(0, \ldots, 0)$. Поэтому пороговый эффект не наблюдается.

Замечание 3. Частный вариант теоремы 1 ранее был доказан Г.В.Балакиным в [3] для случая $R=1$ и функции от двух булевых переменных $\varphi_{1}=\varphi\left(y_{1}, y_{2}\right)$. 
Пример. Рассмотрим пример системы, для которой пороговый эффект имеет место, и вычислим величину $\gamma^{*}$. Пусть $q=2, R=2$,

$$
\varphi_{r}=\varphi_{r}\left(y_{1}, \ldots, y_{d}\right)=y_{1} \oplus \ldots \oplus y_{d} \oplus(r-1), \quad r=1,2,
$$

где $d \geqslant 1$, знак $\oplus$ означает сложение в $G F(2)$. Положим $r^{\prime}=r-1$. Для величин (6) имеем

$$
L_{\varphi_{r}}\left(\alpha ; d_{0}, d_{1}\right)=\left(\begin{array}{c}
d \\
d_{1}
\end{array}\right) I\left\{d_{1} \equiv r^{\prime} \oplus \alpha \bmod 2\right\},
$$

где $I\{E\}$ обозначает индикатор события $E$. Поэтому функция (10) представляется формулой

$$
f\left(z_{0}, z_{1}\right)=\tau(1) \ln \phi_{1}\left(z_{0}, z_{1}\right)+\tau(2) \ln \phi_{2}\left(z_{0}, z_{1}\right),
$$

где

$$
\begin{gathered}
\phi_{r}\left(z_{0}, z_{1}\right)=\sum_{\alpha \in\{0,1\}} P\left\{a_{t, r}=\alpha\right\} \times \\
\times \sum_{v \geqslant 0:\left(r^{\prime} \oplus \alpha\right)+2 v \leqslant d}\left(\begin{array}{c}
d \\
\left(r^{\prime} \oplus \alpha\right)+2 v
\end{array}\right) z_{1}^{\left(r^{\prime} \oplus \alpha\right)+2 v} z_{0}^{d-\left(\left(r^{\prime} \oplus \alpha\right)+2 v\right) .}
\end{gathered}
$$

Положим

$$
\mathbf{P}\left\{a_{t, r}=0\right\}=\mathbf{P}\left\{a_{t, r}=1\right\}=1 / 2, \quad r=1,2 .
$$

В этом случае

$$
\left.\phi_{r}\left(z_{0}, z_{1}\right)\right|_{z_{0}+z_{1}=1}=1 / 2,\left.\quad f\left(z_{0}, z_{1}\right)\right|_{z_{0}+z_{1}=1}=-\ln 2,
$$

и величина $\gamma^{*}$ принимает значение

$$
\gamma^{*}=\sup _{z_{0}, z_{1}: z_{0}+z_{1}=1} \frac{H\left(z_{0}, z_{1}\right)}{f\left(z_{0}, z_{1}\right)}=\sup _{z_{0}, z_{1}: z_{o}+z_{1}=1} \frac{-z_{0} \ln z_{0}-z_{1} \ln z_{1}}{\ln 2}=1 .
$$

Доказательство теоремы. Обозначим через $\pi_{r}(x)$ вероятность события, которое состоит в том, что вектор $x=\left(x_{1}, \ldots, x_{n}\right)$ удовлетворяет уравнению с функцией $\varphi_{r}$. Пусть вектор $x$ содержит $n_{i}(x)$ координат, принимающих значение $i \in V_{q}$. Всем векторам $x$, имеющим одинаковый набор чисел

$$
n_{i}(x)=n_{i}, \quad i \in V_{q},
$$


соответствует одинаковая вероятность

$$
\pi_{r}(x)=P_{r}\left[n_{0}, \ldots, n_{q-1}\right] .
$$

Количество векторов $x$ с фиксированным набором чисел (15) равно

$$
\frac{n !}{n_{0} ! \cdot \ldots \cdot n_{q-1} !}
$$

Поэтому

$$
\begin{gathered}
\mathbf{E} \xi=\sum_{x \in\{0, \ldots, q-1\}^{n}} \prod_{r=1}^{R}\left(\pi_{r}(x)\right)^{T(r)}= \\
=\sum_{\substack{n_{0}, \ldots, n_{q-1} \\
n_{0}+\ldots+n_{q-1}=n}} \frac{n !}{n_{0} ! \cdot \ldots \cdot n_{q-1} !} \prod_{r=1}^{R}\left(P_{r}\left[n_{0}, \ldots, n_{q-1}\right]\right)^{T(r)} .
\end{gathered}
$$

Для вероятности (16) имеем

$$
P_{r}\left[n_{0}, \ldots, n_{q-1}\right]=\sum_{\alpha \in A_{\varphi_{r}}} P_{r}(\alpha) P_{r}\left[n_{0}, \ldots, n_{q-1} \mid a_{t, r}=\alpha\right]
$$

где (с учетом формулы (2))

$$
\begin{aligned}
& P_{r}\left[n_{0}, \ldots, n_{q-1} \mid a_{t, r}=\alpha\right]=\left[\left(\begin{array}{c}
n \\
d(r)
\end{array}\right) \frac{d(r) !}{\left|\Sigma_{\varphi_{r}}\right|}\right]^{-1} \times \\
\times & \sum_{\substack{d_{0}, \ldots, d_{q-1} \\
d_{0}+\ldots+d_{q-1}=d(r)}}\left(\frac{\prod_{s=0}^{q-1} d_{s} !}{\left|\Sigma_{\varphi_{r}}\right|} L_{\varphi_{r}}\left(\alpha ; d_{0}, \ldots, d_{q-1}\right)\right) \prod_{i=0}^{q-1}\left(\begin{array}{c}
n_{i} \\
d_{i}
\end{array}\right)
\end{aligned}
$$

и $L_{\varphi_{r}}\left(\alpha ; d_{0}, \ldots, d_{q-1}\right)$ - величина, заданная равенством $(6)$.

Выберем некоторое число $\varepsilon, 0<\varepsilon<1$, и разобьем сумму (17) на две части

$$
\mathbf{E} \xi=\sum^{*}+\sum^{* *}
$$

полагая

$$
\sum^{*}=\sum_{\substack{n_{0}+\ldots+n_{q-1}=n \\ n_{i} \geqslant \varepsilon n, i=0, q-1}} \frac{n !}{n_{0} ! \cdot \ldots \cdot n_{q-1} !} \prod_{r=1}^{R}\left(P_{r}\left[n_{0}, \ldots, n_{q-1}\right]\right)^{T(r)}
$$




$$
\sum^{* *}=\sum_{\substack{n_{0}+\ldots+n_{q-1}=n \\ \exists i: n_{i}<\varepsilon n}} \frac{n !}{n_{0} ! \cdot \ldots \cdot n_{q-1} !} \prod_{r=1}^{R}\left(P_{r}\left[n_{0}, \ldots, n_{q-1}\right]\right)^{T(r)} .
$$

В области

$$
\sum_{i=0}^{q-1} n_{i}=n, \quad n_{i} \geqslant \varepsilon n, \quad i=\overline{0, q-1}
$$

значений вектора $\left(n_{0}, \ldots, n_{q-1}\right)$ выполняются соотношения $n_{0}, \ldots, n_{q-1} \rightarrow \infty$, и из формул (18), (19) с учетом оценок

$$
\left(\begin{array}{c}
n \\
d(r)
\end{array}\right)=\frac{n^{d(r)}}{d(r) !}(1+o(1)), \quad\left(\begin{array}{c}
n_{i} \\
d_{i}
\end{array}\right)=\frac{n_{i}^{d_{i}}}{d_{i} !}(1+o(1)), \quad i=1, \ldots, q-1,
$$

получаем

$$
\begin{gathered}
P_{r}\left[n_{0}, \ldots, n_{q-1}\right]= \\
=(1+o(1)) \sum_{\alpha \in A_{\varphi_{r}}} P_{r}(\alpha) \times \sum_{\begin{array}{c}
d_{0}, \ldots, d_{q-1} \\
d_{0}+\ldots+d_{q-1}=d(r)
\end{array}} L_{\varphi_{r}}\left(\alpha ; d_{0}, \ldots, d_{q-1}\right) \prod_{i=0}^{q-1}\left(\frac{n_{i}}{n}\right)^{d_{i}}= \\
=(1+o(1)) \sum_{\substack{d_{0}, \ldots, d_{q-1} \\
d_{0}+\ldots+d_{q-1}=d(r)}} L_{\varphi_{r}}\left(d_{0}, \ldots, d_{q-1}\right) \prod_{i=0}^{q-1}\left(\frac{n_{i}}{n}\right)^{d_{i}},
\end{gathered}
$$

где $L_{\varphi_{r}}\left(d_{0}, \ldots, d_{q-1}\right)$ - величина, заданная формулой $(7)$.

Далее, опять рассматривая формулу (17), получаем

$$
\begin{gathered}
\prod_{r=1}^{R}\left(P_{r}\left[n_{0}, \ldots, n_{q-1}\right]\right)^{T(r)}=\exp \left\{T \sum_{r=1}^{R} \tau(r) \ln P_{r}\left[n_{0}, \ldots, n_{q-1}\right]\right\}= \\
=\exp \left\{T f\left(\frac{n_{0}}{n}, \ldots, \frac{n_{q-1}}{n}\right)+o(T)\right\} .
\end{gathered}
$$

Вместе с этим, при условии (21) согласно формуле Стирлинга (см. стр. 67 в [6])

$$
\begin{gathered}
\frac{n !}{n_{0} ! \cdot \ldots \cdot n_{q-1} !}= \\
=(1+o(1))(2 \pi n)^{(1-q) / 2}\left(\prod_{i=0}^{q-1} \frac{n_{i}}{n}\right)^{-1 / 2} \exp \left\{-n H\left(\frac{n_{0}}{n}, \ldots, \frac{n_{q-1}}{n}\right)\right\},
\end{gathered}
$$


где $H\left(z_{0}, \ldots, z_{q-1}\right)$ - величина, определенная формулой $(11)$. Поэтому с учетом (10)

$$
\begin{gathered}
\sum^{*}=(1+o(1)) \sum_{\substack{n_{0}+\ldots+n_{q-1}=n \\
\left(n_{i} / n\right) \geqslant \varepsilon, i=\overline{0, q-1}}}(2 \pi n)^{(1-q) / 2}\left(\prod_{i=0}^{q-1} \frac{n_{i}}{n}\right)^{-1 / 2} \times \\
\times \exp \left\{-n R\left(T ; \frac{n_{0}}{n}, \ldots, \frac{n_{q-1}}{n}\right)\right\},
\end{gathered}
$$

где (полагаем $\left.H=H\left(\frac{n_{0}}{n}, \ldots, \frac{n_{q-1}}{n}\right), f=f\left(\frac{n_{0}}{n}, \ldots, \frac{n_{q-1}}{n}\right)\right)$

$$
\begin{gathered}
R\left(T ; \frac{n_{0}}{n}, \ldots, \frac{n_{q-1}}{n}\right)= \\
=H-\frac{T}{n}(f+o(1))=H-\frac{T}{n} f(1+o(1))=f\left[\frac{H}{f}-\frac{T}{n}(1+o(1))\right] .
\end{gathered}
$$

При условии

$$
\frac{T}{n} \geqslant \gamma^{*}+\delta, \quad \delta>0,
$$

каждому слагаемому в оценке (24), начиная с некоторых значений $n, T$, соответствует оценка (с учетом (25), (12) и неравенств $\left.f\left(\frac{n_{0}}{n}, \ldots, \frac{n_{q-1}}{n}\right)<0\right)$

$$
R\left(T ; \frac{n_{0}}{n}, \ldots, \frac{n_{q-1}}{n}\right) \geqslant-f\left(\frac{n_{0}}{n}, \ldots, \frac{n_{q-1}}{n}\right)\left(\delta+w_{n}\right),
$$

где $w_{n}=o(1)$ при $n \rightarrow \infty$. При этом согласно (14) существует такая постоянная величина $\delta^{\prime}>0$, что

$$
-f\left(\frac{n_{0}}{n}, \ldots, \frac{n_{q-1}}{n}\right) \geqslant \delta^{\prime}>0 .
$$

Поэтому из (27) следует оценка

$$
R\left(T ; \frac{n_{0}}{n}, \ldots, \frac{n_{q-1}}{n}\right) \geqslant \delta^{\prime}\left(\delta+w_{n}\right)>0 .
$$

Следовательно, при условии (26)

$$
\sum^{*} \rightarrow 0
$$

Пусть теперь

$$
\frac{T}{n} \leqslant \gamma^{*}-\delta, \quad \delta>0
$$


Функция

$$
G\left(z_{0}, \ldots, z_{q-1}\right)=\frac{H\left(z_{1}, \ldots, z_{q-1}\right)}{f\left(z_{1}, \ldots, z_{q-1}\right)}
$$

непрерывна в каждой точке множества

$$
Z=\left\{\left(z_{0}, \ldots, z_{q-1}\right): z_{0}+\ldots+z_{q-1}=1, \quad z_{i} \geqslant 0, \quad i \in V_{q}\right\} .
$$

Поэтому при $0<\delta^{\prime \prime}<\delta$ и достаточно малом $\varepsilon>0$ найдется такое слагаемое в правой части (24), что

$$
G\left(\frac{n_{0}}{n}, \ldots, \frac{n_{q-1}}{n}\right) \geqslant \gamma^{*}-\delta^{\prime \prime}
$$

и с учетом символического соотношения $\frac{T}{n}(1+o(1))=\frac{T}{n}+o(1)$ при $0 \leqslant \frac{T}{n} \leqslant \gamma^{*}-\delta($ см. $(25))$

$$
\begin{aligned}
R\left(T ; \frac{n_{0}}{n}, \ldots, \frac{n_{q-1}}{n}\right)= & f\left(\frac{n_{0}}{n}, \ldots, \frac{n_{q-1}}{n}\right)\left(G\left(\frac{n_{0}}{n}, \ldots, \frac{n_{q-1}}{n}\right)-\frac{T}{n}+o(1)\right) \leqslant \\
& \leqslant-\delta^{\prime}\left(\delta-\delta^{\prime \prime}+w_{n}^{\prime}\right)<0,
\end{aligned}
$$

где величина $\delta^{\prime}$ берется из неравенства (28) и $w_{n}^{\prime}=o(1)$ при $n \rightarrow \infty$. Следовательно, если выполняется условие $(29)$, то $\sum^{*} \rightarrow \infty$.

Для завершения доказательства теоремы осталось показать, что при условии (26)

$$
\sum^{* *} \rightarrow 0
$$

Положим

$$
\begin{gathered}
N_{\varepsilon}\left(i_{1}, \ldots, i_{k}\right)=\left\{\left(n_{0}, \ldots, n_{q-1}\right): \sum_{i=0}^{q-1} n_{i}=n ; n_{i_{j}}<\varepsilon n, j=\overline{1, k} ;\right. \\
\left.n_{i} \geqslant \varepsilon n, i \in\{0, \ldots, q-1\} \backslash\left\{i_{1}, \ldots, i_{k}\right\}\right\}
\end{gathered}
$$

и представим $\sum^{* *}$ в следующем виде:

$$
\begin{gathered}
\sum^{* *}=\sum_{k=1}^{q-1} \sum_{0 \leqslant i_{1}<\ldots<i_{k} \leqslant q-1} \sum_{\left(n_{0}, \ldots, n_{q-1}\right) \in N_{\varepsilon}\left(i_{1}, \ldots, i_{k}\right)} \frac{n !}{n_{0} ! \cdot \ldots \cdot n_{q-1} !} \times \\
\times \prod_{r=1}^{R}\left(P_{r}\left[n_{0}, \ldots, n_{q-1}\right]\right)^{T(r)} .
\end{gathered}
$$


Сначала оценим комбинаторный множитель в правой части (30). При $\left(n_{0}, \ldots, n_{q-1}\right) \in N_{\varepsilon}\left(i_{1}, \ldots, i_{k}\right)$ имеем

$$
\frac{n !}{n_{0} ! \ldots n_{q-1} !}=\frac{\left(\sum_{j=1}^{k} n_{i_{j}}\right) !}{\prod_{j=1}^{k} n_{i_{j}} !} \cdot\left(\begin{array}{c}
n \\
\sum_{j=1}^{k} n_{i_{j}}
\end{array}\right) \cdot \frac{\left(n-\sum_{j=1}^{k} n_{i_{j}}\right) !}{\prod_{\nu \notin\left\{i_{1}, \ldots, i_{k}\right\}} n_{\nu} !} .
$$

Для оценки каждого из трех множителей в правой части (31) воспользуемся общим неравенством вида

$$
\frac{m !}{m_{1} ! \ldots m_{s} !} \leqslant \frac{m^{m}}{m_{1}^{m_{1}} \ldots m_{s}^{m_{s}}}=\exp \left\{-m H\left(\frac{m_{1}}{m}, \ldots, \frac{m_{s}}{m}\right)\right\}
$$

где $s \geqslant 2, m_{1}+\ldots+m_{s}=m$, следующим из оценки $\left(\begin{array}{l}n \\ r\end{array}\right) \leqslant \frac{n !}{r !(n-r) !}[7$, с. 27]. Кроме того, для оценки первого множителя применим неравенство

$$
-H\left(\frac{m_{1}}{m}, \ldots, \frac{m_{s}}{m}\right) \leqslant-H\left(\frac{1}{s}, \ldots, \frac{1}{s}\right)=\ln s,
$$

которое отражает экстремальное свойство энтропии равномерного распределения на конечном множестве.

Используя (32), (33), для первого множителя в правой части (31) получаем оценку

$$
\frac{\left(\sum_{j=1}^{k} n_{i_{j}}\right) !}{\prod_{j=1}^{k} n_{i_{j}} !} \leqslant \exp \{k \cdot n \varepsilon \ln k\}
$$

Для второго множителя с помощью (32) находим, что при $k \varepsilon<1$

$$
\left(\begin{array}{c}
n \\
\sum_{j=1}^{k} n_{i_{j}}
\end{array}\right) \leqslant \exp \{-n H(k \varepsilon, 1-k \varepsilon)\}
$$

где $-H(k \varepsilon, 1-k \varepsilon)$ есть энтропия биномиального распределения $B(k \varepsilon)$.

Перед оценкой третьего множителя отметим, что

$$
0 \leqslant \sum_{j=1}^{k} n_{i_{j}} \leqslant n \varepsilon \cdot k
$$

Пусть $n_{i_{j}}=\theta_{j} n$, тогда можно положить

$$
\sum_{j=1}^{k} n_{i_{j}}=n \theta \cdot k, \quad \theta=\left(\theta_{1}+\ldots+\theta_{k}\right) / k, \quad 0 \leqslant \theta \leqslant \varepsilon .
$$


Используя (32), (34), получаем

$$
\begin{gathered}
\frac{\left(n-\sum_{j=1}^{k} n_{i_{j}}\right) !}{\prod_{\nu \notin\left\{i_{1}, \ldots, i_{k}\right\}} n_{\nu} !} \leqslant \exp \left\{-n(1-k \theta) H\left(z_{0}(\theta), \ldots, z_{q-1}(\theta)\right)\right\} \leqslant \\
\leqslant \exp \left\{-n H\left(z_{0}(\theta), \ldots, z_{q-1}(\theta)\right)\right\},
\end{gathered}
$$

где

$$
z_{i}(\theta)=\frac{n_{i}}{n(1-k \theta)} I\left\{i \in\{0, \ldots, q-1\} \backslash\left\{i_{1}, \ldots, i_{k}\right\}\right\},
$$

$I\{E\}-$ индикатор события $E$.

Таким образом получаем, что при $\left(n_{0}, \ldots, n_{q-1}\right) \in N_{\varepsilon}\left(i_{1}, \ldots, i_{k}\right)$

$$
\frac{n !}{n_{0} ! \ldots n_{q-1} !} \leqslant \exp \left\{n\left(-H\left(z_{0}(\theta), \ldots, z_{q-1}(\theta)\right)+C(\varepsilon)\right)\right\},
$$

где $C(\varepsilon)=k \varepsilon \ln k-H(k \varepsilon, 1-k \varepsilon)$. Отметим, что при $\varepsilon \rightarrow 0$

$$
C(\varepsilon)=O(\varepsilon \ln \varepsilon) .
$$

Теперь оценим величины $P_{r}\left[n_{0}, \ldots, n_{q-1}\right]$. Аналогично (22) при $\left(n_{0}, \ldots, n_{q-1}\right) \in N_{\varepsilon}\left(i_{1}, \ldots, i_{k}\right)$ получаем, что

$$
P_{r}\left[n_{0}, \ldots, n_{q-1}\right] \leqslant\left(1+w_{n, r}\right) \sum_{d_{0}+\ldots+d_{q-1}=d(r)} \prod_{i=0}^{q-1}\left(\frac{n_{i}}{n}\right)^{d_{i}} L_{\varphi_{r}}\left(d_{0}, \ldots, d_{q-1}\right),
$$

где $w_{n, r}=o(1)$ при $n \rightarrow \infty$. Отсюда, из неравенств (см. (35))

$$
\begin{gathered}
\left(\frac{n_{i}}{n}\right)^{d_{i}} \leqslant\left(z_{i}(\theta)\right)^{d_{i}}, \quad i \in\{0, \ldots, q-1\} \backslash\left\{i_{1}, \ldots, i_{k}\right\}, \\
\left(\frac{n_{i}}{n}\right)^{d_{i}} \leqslant \varepsilon^{d_{i}}, \quad i \in\left\{i_{1}, \ldots, i_{k}\right\}
\end{gathered}
$$

и определения множества $N_{\varepsilon}\left(i_{1}, \ldots, i_{k}\right)$ следует, что

$$
P_{r}\left[n_{0}, \ldots, n_{q-1}\right] \leqslant\left(1+w_{n, r}\right)\left[D_{r}\left(\theta ; n_{0}, \ldots, n_{q-1}\right)+K^{\prime}(\varepsilon)\right],
$$

где $K^{\prime}(\varepsilon)=O(\varepsilon)$ при $\varepsilon \rightarrow 0$, и

$$
D_{r}\left(\theta ; n_{0}, \ldots, n_{q-1}\right)=\sum_{\substack{d_{0}+\ldots+d_{q-1}=d(r) \\ d_{i}=0, i \in\left\{i_{1}, \ldots, i_{k}\right\}}} \prod_{i=0}^{q-1}\left(z_{i}(\theta)\right)^{d_{i}} L_{\varphi_{r}}\left(d_{0}, \ldots, d_{q-1}\right) .
$$


Заметим, что согласно определениям (6), (7) и условию $\mathbf{A}$

$$
L_{\varphi_{r}}\left(d_{0}, \ldots, d_{q-1}\right) \geqslant \frac{d(r) !}{d_{0} ! \ldots d_{q-1} !} \min _{\alpha \in A_{\varphi_{r}}} P_{r}(\alpha)>0 .
$$

Из (39), (40) и равенств

$$
\sum_{\substack{d_{0}+\ldots+d_{q-1}=d(r) \\ d_{i}=0, i \in\left\{i_{1}, \ldots, i_{k}\right\}}} \prod_{i=0}^{q-1}\left(z_{i}(\theta)\right)^{d_{i}} \frac{d(r)}{d_{0} ! \ldots d_{q-1} !}=\left(z_{0}(\theta)+\ldots+z_{q-1}(\theta)\right)^{d(r)}=1
$$

получаем, что

$$
D_{r}\left(\theta ; n_{0}, \ldots, n_{q-1}\right) \geqslant \min _{\alpha \in A_{\varphi_{r}}} P_{r}(\alpha)>0 .
$$

С учетом (41) соотношение (38) можно переписать в виде

$$
P_{r}\left[n_{0}, \ldots, n_{q-1}\right] \leqslant\left(1+w_{n, r}\right) D_{r}\left(\theta ; n_{0}, \ldots, n_{q-1}\right)\left(1+K^{\prime \prime}(\varepsilon)\right),
$$

где $K^{\prime \prime}(\varepsilon)=K^{\prime}(\varepsilon) / D_{r}\left(n_{0}, \ldots, n_{q-1}\right)=O(\varepsilon)$. В силу равенства (см. (10), (39))

$$
f\left(z_{0}(\theta), \ldots, z_{q-1}(\theta)\right)=\sum_{r=1}^{R} \tau(r) \ln D_{r}\left(\theta ; n_{0}, \ldots, n_{q-1}\right)
$$

и соотношения (14)

$$
\begin{gathered}
\prod_{r=1}^{R}\left(P_{r}\left[n_{0}, \ldots, n_{q-1}\right]\right)^{T(r)}=\exp \left\{T \sum_{r=1}^{R} \tau(r) \ln P_{r}\left[n_{0}, \ldots, n_{q-1}\right]\right\} \leqslant \\
\leqslant \exp \left\{T f\left(z_{0}(\theta), \ldots, z_{q-1}(\theta)\right)\left(1+K^{\prime \prime \prime}(\varepsilon)+w_{n}\right)\right\},
\end{gathered}
$$

где $K^{\prime \prime \prime}(\varepsilon)=O(\varepsilon)$ при $\varepsilon \rightarrow 0$, а $w_{n}=o(1)$ при $n \rightarrow \infty$.

Следовательно, для внутренней суммы в представлении (30) справедлива оценка (см. (36) и (42)):

$$
\begin{gathered}
\sum_{\left(n_{0}, \ldots, n_{q-1}\right) \in N_{\varepsilon}\left(i_{1}, \ldots, i_{k}\right)} \frac{n !}{n_{0} ! \cdot \ldots \cdot n_{q-1} !} \prod_{r=1}^{R}\left(P_{r}\left[n_{0}, \ldots, n_{q-1}\right]\right)^{T(r)} \leqslant \\
\leqslant \sum_{\left(n_{0}, \ldots, n_{q-1}\right) \in N_{\varepsilon}\left(i_{1}, \ldots, i_{k}\right)} \exp \left\{-n R\left(T ; z_{0}(\theta), \ldots, z_{q-1}(\theta)\right)\right\},
\end{gathered}
$$

где

$$
R\left(T ; z_{0}(\theta), \ldots, z_{q-1}(\theta)\right)=
$$




$$
\begin{gathered}
=H\left(z_{0}(\theta), \ldots, z_{q-1}(\theta)\right)-C(\varepsilon)-\frac{T}{n} f\left(z_{0}(\theta), \ldots, z_{q-1}(\theta)\right)\left(1+K^{\prime \prime \prime}(\varepsilon)+w_{n}\right)= \\
=f\left[\frac{H}{f}-\frac{T}{n}\left(1+K^{\prime \prime \prime}(\varepsilon)+w_{n}\right)-\frac{C(\varepsilon)}{f}\right] .
\end{gathered}
$$

Здесь использованы обозначения $H=H\left(z_{0}(\theta), \ldots, z_{q-1}(\theta)\right), f=$ $f\left(z_{0}(\theta), \ldots, z_{q-1}(\theta)\right)$. Согласно $(12)$ (учитываем, что $0 \leqslant z_{0}(\theta), \ldots, 0 \leqslant$ $\left.z_{0}(\theta), z_{0}(\theta)+\ldots+z_{q-1}(\theta)=1\right)$

$$
\frac{H}{f}=\frac{H\left(z_{0}(\theta), \ldots, z_{q-1}(\theta)\right)}{f\left(z_{0}(\theta), \ldots, z_{q-1}(\theta)\right)} \leqslant \gamma^{*} .
$$

Поэтому величина (44) при $T / n>\gamma^{*}+\delta$ и достаточно малом $\varepsilon$ больше некоторой положительной константы $\delta(\varepsilon)$. Эта оценка величины $(44)$ равномерна относительно наборов $\left(n_{0}, \ldots, n_{q-1}\right) \in N_{\varepsilon}\left(i_{1}, \ldots, i_{k}\right)$. Теперь с учетом неравенства $\left|N_{\varepsilon}\left(i_{1}, \ldots, i_{k}\right)\right|<n^{q}$ получаем

$$
\sum_{\left(n_{0}, \ldots, n_{q-1}\right) \in N_{\varepsilon}\left(i_{1}, \ldots, i_{k}\right)} \frac{n !}{n_{0} ! \cdot \ldots \cdot n_{q-1} !} \prod_{r=1}^{R}\left(P_{r}\left[n_{0}, \ldots, n_{q-1}\right]\right)^{T(r)} \rightarrow 0 .
$$

Следовательно (см. (30)), $\sum^{* *} \rightarrow 0$. Теорема доказана.

Автор признателен В.Г. Михайлову за полезные замечания.

\section{Список литературы}

[1] Балакин Г.В., Колчин В.Ф., Хохлов В.И., "Гиперциклы в случайном гиперграфе", Дискретная математика, 3:3 (1991), 102-108.

[2] Колчин В.Ф., Хохлов В.И., "Пороговый эффект для систем линейных уравнений специального вида", Дискретная математика, 7:4 (1995), 29-39.

[3] Балакин Г.В., "Графы систем двучленных уравнений с булевыми неизвестными", Теория вероятностей и ее применения, 40:2 (1995), 241-259.

[4] Колчин В.Ф., "О пороговом эффекте для систем случайных уравнений”, Трудъ по дискретной математике, 2 (1998), 183-190.

[5] Копытцев В.А., "Предельные теоремы для числа решений системы случайных уравнений”, Теория вероятностей и ее применения, 45:1 (2000), 52-72.

[6] Феллер В., Введение в теорию вероятностей и ее приложения, m. 1, М. : Мир, 1964.

[7] Риордан Дж., Введение в комбинаторный анализ, М. : ИЛ, 1963, 288 с. 